VOL. $71(2005) \quad$ [285-303]

\title{
EXISTENCE CONDITIONS IN GENERAL QUASIMONOTONE VARIATIONAL INEQUALITIES
}

\author{
D. Aussel and D.T. LuC
}

In this paper we study a general variational inequality model with set-valued quasimonotone operators, a model which includes several variational inequalities and equilibrium problems. We establish unifying conditions for existence of solutions in a topological vector space setting. Applications to parametric equilibrium models and to a contact problem are given.

\section{INTRODUCTION}

Throughout this paper we shall make use of the following notations. $X$ and $Y$ are real Hausdorff topological vector spaces, $K$ is a nonempty subset of $X, \phi$ is a real function on $Y \times K$ which is sometimes called a coupling function between $Y$ and $K$, and $T$ is a set-valued operator from $K$ to $Y$. The topological dual of $X$ is denoted by $X^{\prime}$ and the pairing function between $X$ and $X^{\prime}$ is written in the form $\left\langle x^{*}, x\right\rangle$ for $x \in X$ and $x^{*} \in X^{\prime}$.

The variational inequality model that we are going to study in this paper is the following:

(V) Find $x_{0} \in K$ such that

$$
\phi\left(x_{0}^{*}, x\right)-\phi\left(x_{0}^{*}, x_{0}\right) \geqslant 0, \forall x \in K, \forall x_{0}^{*} \in T\left(x_{0}\right) .
$$

This model is quite simple, albeit general and includes several variational inequalities and equilibrium problems. Here are some of them that can be found in $[4,7,10,22,25]$.

A. The standard variational inequality introduced by Stampacchia: Find $x_{0} \in K$ such that

$$
\left\langle f\left(x_{0}\right), x-x_{0}\right\rangle \geqslant 0, \forall x \in K,
$$

where $f$ is an operator from $K$ to $X^{\prime}$. This problem is a particular case of model $(V)$ when $T(x)=f(x), Y=X^{\prime}$ and $\phi\left(x^{*}, x\right)=\left\langle x^{*}, x\right\rangle$.

B. The mixed variational inequality problem: Find $x_{0} \in K$ such that

$$
\left\langle x_{0}^{*}, x-x_{0}\right\rangle+h(x)-h\left(x_{0}\right) \geqslant 0, \forall x \in K, \forall x_{0}^{*} \in T\left(x_{0}\right),
$$

Received 9th November, 2004

Copyright Clearance Centre, Inc. Serial-fee code: 0004-9727/05 \$A2.00+0.00. 
where $h$ is a real function on $K$. This problem is obtained from $(V)$ by setting $Y=X^{\prime}$ and $\phi\left(x^{*}, x\right)=\left\langle x^{*}, x\right\rangle+h(x)$.

C. The general variational inequality problem: Find $x_{0} \in X$ with $g\left(x_{0}\right) \in D$ such that

$$
\left\langle f\left(x_{0}\right), g(x)-g\left(x_{0}\right)\right\rangle \geqslant 0, \forall x \in X \text { with } g(x) \in D,
$$

where $g$ is a transformation on $X$. To derive this problem from $(V)$ it suffices to set $Y=X^{\prime}, K=\{x \in X: g(x) \in D\}, \phi\left(x^{*}, x\right)=\left\langle x^{*}, g(x)\right\rangle$ and $T(x)=f(x)$.

D. The equilibrium problem: Find $x_{0} \in K$ such that

$$
f\left(x_{0}, x\right)+h(x)-h\left(x_{0}\right) \geqslant 0, \forall x \in K
$$

where $f$ is a real-valued function on $K \times K$ with $f(x, x)=0$ for every $x \in K$ and $h$ is a real-valued function on $K$. To express this problem in form of variational model $(V)$ it suffices to set $Y=X, T(x)=x$ and $\phi\left(x^{*}, x\right)=f\left(x^{*}, x\right)+h(x)$ for $x^{*} \in Y$ and $x \in K$.

E. The parametric equilibrium problem: Find $x_{0} \in K$ such that

$$
f\left(x_{0}, x\right)+h(z, x)-h\left(z, x_{0}\right) \geqslant 0, \forall x \in K, z \in Z,
$$

where $Z$ is a nonempty set, $f$ is a real-valued function on $K \times K$ with $f(x, x)=0$ for every $x \in K$ and $h$ is a real-valued function on $Z \times K$. To formulate this problem in form of variational model $(V)$ it suffices to set $Y=K \times Z, T(x)=\{x\} \times Z$ and $\phi((y, z), x)=f(y, x)+h(z, x)$ for $(y, z) \in Y$ and $x \in K$.

Together with model $(V)$ we are also interested in the following auxiliary models:

$\left(V_{0}\right) \quad$ Find $x_{0} \in K$ such that for some $x_{0}^{*} \in T\left(x_{0}\right)$,

$$
\phi\left(x_{0}^{*}, x\right)-\phi\left(x_{0}^{*}, x_{0}\right) \geqslant 0, \forall x \in K
$$

$\left(V_{w}\right) \quad$ Find $x_{0} \in K$ such that for every $x \in K$, there is some $x_{0}^{*} \in T\left(x_{0}\right)$ verifying

$$
\phi\left(x_{0}^{*}, x\right)-\phi\left(x_{0}^{*}, x_{0}\right) \geqslant 0
$$

and the so-called Minty variational inequality.

(M) Find $x_{0} \in K$ such that

$$
\phi\left(x^{*}, x\right)-\phi\left(x^{*}, x_{0}\right) \geqslant 0, \forall x \in K, \forall x^{*} \in T(x)
$$

We refer the interested reader to $[4,8,10,16,22,23,26]$ and many references given therein for historical developments, general theory and applications of variational inequalities.

The aim of the present paper is to establish sharp criteria for existence of solutions of the above unifying variational problems when the operator $T$ is quasimonotone. Our 
results are proven by elementary techniques and at the same time enjoy certain degree of generality which makes them applicable to a number of variational inequalities and equilibrium models. By this they unify and strengthen several theorems of recent works on the topics, including $[2,4,5,7,9,21,25,27]$ and some others.

The paper is organised as follows. In the next section we introduce new concepts of generalised monotonicity and generalised continuity of set-valued operators with respect to the function $\phi$. These include almost all particular cases studied in the literature on variational inequalities. In Section 3 relationships between solutions sets of the models $(V)-(M)$ are presented. Section 4 is devoted to sufficient conditions for existence of solutions of the above models. An application to a generalised complementarity problem is considered. Other applications are given respectively in Sections 5 and 6 for equilibrium problems and a frictionless contact problem.

\section{Generalised monotonicity and GENERALISED CONTINUITY}

Following the notations of the introduction $T$ is a set-valued operator from $K$ to $Y$.

Definition 2.1: The operator $T$ is said to be $\phi$-quasimonotone (respectively, weakly $\phi$-quasimonotone) at $x \in K$ if strict inequality

$$
\phi\left(y^{*}, x\right)-\phi\left(y^{*}, y\right)>0, \text { for some } y \in K \text { and for some } y^{*} \in T(y)
$$

(respectively, for some $y \in K$ and for all $y^{*} \in T(y)$ ) implies

$$
\phi\left(x^{*}, x\right)-\phi\left(x^{*}, y\right) \geqslant 0, \text { for every } x^{*} \in T(x) .
$$

When inequality

$$
\phi\left(y^{*}, x\right)-\phi\left(y^{*}, y\right) \geqslant 0, \text { for some } y \in K \text { and for some } y^{*} \in T(y)
$$

(respectively, for some $y \in K$ and for all $y^{*} \in T(y)$ ) implies (2), we say that $T$ is $\phi$-pseudomonotone (respectively, weakly $\phi$-pseudomonotone) at $x$.

When $Y=X^{\prime}$ and $\phi$ is the pairing function $(.,$.$) between X$ and $X^{\prime}$, and when $T$ is single-valued, the above definition reduces to the concept of quasimonotone operators and pseudomonotone operators that were first introduced by Karamardian [13], Karamardian and Schaible [14] for vector-valued functions. Extensions for set-valued operators can be found in $[18,19,20]$ (see also $[1,5,9,24])$. We notice that the notion of $h$-quasimonotonicity (respectively, $h$-quasi-semi-monotonicity) introduced in [4] corresponds to $\phi$-pseudomonotonicity (respectively, weak $\phi$-pseudomonotonicity) when $\phi\left(x^{*}, x\right)$ is given by $\left\langle x^{*}, x\right\rangle+h(x)$.

DEFinition 2.2: The operator $T$ is said to be properly $\phi$-quasimonotone on $K$ if for every convex combination $x$ of $x_{1}, \ldots, x_{n} \in K$, there exists some $i \in\{1, \ldots, n\}$ such that $\phi\left(x_{i}^{*}, x_{i}\right)-\phi\left(x_{i}^{*}, x\right) \geqslant 0$ for all $x_{i}^{*} \in T\left(x_{i}\right)$. 
The notion of proper quasimonotonicity was introduced by Daniilidis and Hadjisavvas in [5] when $\phi$ is given by $\langle.,$.$\rangle . An earlier version under the name of diagonal qua-$ siconcavity was introduced in [28]. It is easy to see that a $\phi$-pseudomonotone operator is properly $\phi$-quasimonotone, which in its turn is $\phi$-quasimonotone. The converse is not always true even when $X=Y^{\prime}$ and $\phi$ is the pairing function $\langle.,$.$\rangle . An equivalent form of$ generalised monotonicity can be given as follows. The operator $T$ is $\phi$-quasimonotone (respectively, weakly $\phi$-quasimonotone) at $x \in K$ if for every $y \in K$, for every (respectively, for some) $y^{*} \in T(y)$ one has

$$
\min \left\{\phi\left(x^{*}, y\right)-\phi\left(x^{*}, x\right), \phi\left(y^{*}, x\right)-\phi\left(y^{*}, y\right)\right\} \leqslant 0
$$

for every $x^{*} \in T(x)$;

$T$ is $\phi$-pseudomonotone (respectively, weakly $\phi$-pseudomonotone) at $x \in K$ if for every $y \in K$, for every (respectively, for some) $y^{*} \in T(y)$ one has

$$
\min \left\{\phi\left(x^{*}, y\right)-\phi\left(x^{*}, x\right), \phi\left(y^{*}, x\right)-\phi\left(y^{*}, y\right)\right\}<0
$$

for all $x^{*} \in T(x)$ with $\phi\left(x^{*}, y\right) \neq \phi\left(x^{*}, x\right)$. Finally $T$ is properly $\phi$-quasimonotone on $K$ if for any convex combination $x$ of elements $x_{1}, \ldots, x_{n} \in K$, one has

$$
\min _{i=1, \ldots, n} \sup _{x_{i}^{*} \in T\left(x_{i}\right)}\left(\phi\left(x_{i}^{*}, y\right)-\phi\left(x_{i}^{*}, x_{i}\right)\right) \leqslant 0 .
$$

When $Y=X^{\prime}$ and $T$ is a subdifferential (in certain sense) of a function, generalised monotonicity is used to characterise generalised convexity of the function (see $[1,20,24]$ for instance). It was noticed in [5] that when $T$ is a subdifferential operator in the sense of Clarke-Rockafellar of a lower semicontinuous function, it is properly quasimonotone if and only if it is quasimonotone, and if and only if the function is quasiconvex.

DefinItion 2.3: We say that $T$ is $\phi$-hemicontinuous (respectively, weakly $\phi$-hemicontinuous) at $x_{0} \in K$ if for every $x \in K$, inequality

$$
\phi\left(x_{t}^{*}, x_{0}+t\left(x-x_{0}\right)\right)-\phi\left(x_{t}^{*}, x_{0}\right) \geqslant 0, \forall x_{t}^{*} \in T\left(x_{0}+t\left(x-x_{0}\right)\right)
$$

for all $t$ sufficiently close to 0 , implies

$$
\phi\left(x_{0}^{*}, x\right)-\phi\left(x_{0}^{*}, x_{0}\right) \geqslant 0, \forall x_{0}^{*} \in T\left(x_{0}\right)
$$

(respectively, for some $x_{0}^{*} \in T\left(x_{0}\right)$ )).

We note that when $Y=X^{\prime}, T\left(x_{0}\right)$ is compact and $\phi$ is given by $\langle.$,$\rangle , weak$ $\phi$-hemicontinuity corresponds to upper sign-continuity of [2]. Below we present some particular cases in which $T$ is $\phi$-hemicontinuous or weakly $\phi$-hemicontinuous. Recall that $T$ is said to be upper semicontinuous (respectively, lower semicontinuous) at $x \in K$ if for every open set $A \subseteq Y$ with $T(x) \subseteq A$ (respectively, $T(x) \cap A \neq \emptyset$ ), there is some neighbourhood $U$ of $x$ such that $T\left(x^{\prime}\right) \subseteq A$ (respectively, $T\left(x^{\prime}\right) \cap A \neq \emptyset$ ) for every $x^{\prime} \in U \cap K$. In our paper this definition is not applied to real-valued functions for which the notions of lower and upper semicontinuities are understood in the usual sense. 
Proposition 2.4. Each of the conditions (i)-(iii) below is sufficient for $T$ to be $\phi$-hemicontinuous:

(i) $\phi$ is continuous, $T$ is lower semicontinuous on segments;

(ii) $Y=X^{\prime}, \phi=\langle.,$.$\rangle and for every x, y \in K$, the function $t \mapsto \inf _{x^{*} \in T(x+t(y-x))}\left(x^{*}, y-x\right\rangle$ is upper semicontinuous on $[0,1]$ at $t=0$;

(iii) $Y=X^{\prime}, \phi=(.,$.$) and T$ is single-valued continuous on segments.

Each of the conditions (iv)-(v) below is sufficient for $T$ to be weakly $\phi$-hemicontinuous:

(iv) $\phi$ is continuous and $T$ is upper semicontinuous on segments and compactvalued;

(v) $Y=X^{\prime}, \phi=\langle.,$.$\rangle and T$ is $w^{*}$-compact-valued and for every $x, y \in K$, the function $t \mapsto \sup _{x^{*} \in T(x+t(y-x))}\left\langle x^{*}, y-x\right\rangle$ is upper semicontinuous on $[0,1]$ at $t=0$.

Proof: Direct verification achieves the proof.

\section{RELATIONShip BETWEen SOLUTION SETS}

In this section we are going to establish some links between the solution sets of variational inequality models $(V),\left(V_{0}\right),\left(V_{w}\right)$ and of the Minty problem.

The solution sets of problems $(V),\left(V_{0}\right),\left(V_{w}\right)$ and $(M)$ are denoted respectively by $S(V), S\left(V_{0}\right), S\left(V_{w}\right)$ and $S(M)$. If there is a neighbourhood $U$ of $x_{0}$ such that the inequality in problem $(M)$ holds for all $x \in K \cap U$, then $x_{0}$ is called a local solution of the Minty variational inequality. The local solution set of $(M)$ is denoted by $L S(M)$.

Proposition 3.1. Assume that $K$ is nonempty and convex. Then one has

(i) $L S(M)=S(M)$ provided that $\phi$ is lower semicontinuous and convex in $x$ and $T$ is weakly $\phi$-pseudomonotone on $K$;

(ii) $L S(M) \subseteq S(V)$ provided that $T$ is $\phi$-hemicontinuous;

(iii) $L S(M) \subseteq S\left(V_{w}\right)$ provided that $T$ locally admits a weakly $\phi$-hemicontinuous suboperator.

Proof: Let us prove (i). The inclusion $S(M) \subseteq L S(M)$ being obvious, we need to prove the converse inclusion only. Let $x_{0} \in L S(M)$, that is, there is some neighbourhood $U$ of $x_{0}$ such that

$$
\phi\left(x^{*}, x\right)-\phi\left(x^{*}, x_{0}\right) \geqslant 0
$$

for every $x \in K \cap U$ and $x^{*} \in T(x)$. Let $y \in K$ be given. There is some $t_{0} \in(0,1)$ such that $x_{0}+t\left(y-x_{0}\right) \in K \cap U$ for $t \in\left[0, t_{0}\right]$. For each $x^{*} \in T\left(x_{0}+t_{0}\left(y-x_{0}\right)\right)$ the convexity 
of the function $t \mapsto \phi\left(x^{*}, x_{0}+t\left(y-x_{0}\right)\right)$ yields

$$
\frac{\phi\left(x^{*}, y\right)-\phi\left(x^{*}, x_{0}+t_{0}\left(y-x_{0}\right)\right)}{1-t_{0}} \geqslant \frac{\phi\left(x^{*}, x_{0}+t_{0}\left(y-x_{0}\right)\right)-\phi\left(x^{*}, x_{0}\right)}{t_{0}}
$$

which together with (6) gives

$$
\phi\left(x^{*}, y\right)-\phi\left(x^{*}, x_{0}+t_{0}\left(y-x_{0}\right)\right) \geqslant 0 .
$$

By the weak $\phi$-pseudomonotonicity of $T$ we derive

$$
\phi\left(y^{*}, y\right)-\phi\left(y^{*}, x_{0}+t_{0}\left(y-x_{0}\right)\right) \geqslant 0
$$

for every $y^{*} \in T(y)$. The same argument shows that (7) is true when $t_{0}$ is replaced by any $t \in\left(0, t_{0}\right)$. The function $\phi\left(y^{*}, x_{0}+t\left(y-x_{0}\right)\right)$ being lower semicontinuous in $t$ we deduce that

$$
\phi\left(y^{*}, y\right)-\phi\left(y^{*}, x_{0}\right) \geqslant 0
$$

for every $y^{*} \in T(y)$. By this, $x_{0} \in S(M)$.

For the second assertion, assume that $x_{0} \in K$ is a local solution of $(M)$, that is (6) is satisfied. Let $y \in K$ be given. For $x_{t}:=x_{0}+t\left(y-x_{0}\right) \in K \cap U$ with $t \in\left[0, t_{0}\right]$ we have

$$
\phi\left(x_{t}^{*}, x_{t}\right)-\phi\left(x_{t}^{*}, x_{0}\right) \geqslant 0
$$

for every $x_{t}^{*} \in T\left(x_{t}\right)$. It follows from the $\phi$-hemicontinuity of $T$ that

$$
\phi\left(x_{0}^{*}, y\right)-\phi\left(x_{0}^{*}, x_{0}\right) \geqslant 0
$$

for every $x_{0}^{*} \in T\left(x_{0}\right)$. This means that $x_{0}$ is a solution of $(V)$.

Finally, if $T$ admits a weakly $\phi$-continuous suboperator $T_{1}$ in a neighbourhood of $x_{0}$, then (8) holds for $t$ sufficiently close to 0 and for all $x_{t}^{*} \in T_{1}\left(x_{t}\right)$. By the weak $\phi-$ hemicontinuity of $T_{1},(9)$ is still true for some $x_{0}^{*} \in T_{1}\left(x_{0}\right) \subseteq T\left(x_{0}\right)$. Consequently, $x_{0}$ is a solution of $\left(V_{w}\right)$.

Proposition 3.2. Assume that $K$ is nonempty and convex. Then the following assertions hold:

(i) $S(V) \subseteq S\left(V_{0}\right) \subseteq S\left(V_{w}\right)$. Equalities are true when $T$ is single-valued;

(ii) Let $x_{0} \in K$ be a solution of $\left(V_{w}\right)$. It is also a solution of $\left(V_{0}\right)$ provided that $T\left(x_{0}\right)$ is compact and convex, and that the function $\left(x^{*}, x\right) \mapsto \phi\left(x^{*}, x\right)$ $-\phi\left(x^{*}, x_{0}\right)$ is convex in $x$ and upper semicontinuous, concave in $x^{*}$.

Proof: The first assertion is obvious. The second assertion is a consequence of the standard minimax theorem [6].

Corollary 3.3. Let $x_{0} \in K$ be a local solution of $(M)$. Then it is a solution of $\left(V_{0}\right)$ when the following conditions hold: 
(a) T admits a suboperator in a neighbourhood of $x_{0}$ which is convex compact-valued and weakly $\phi$-hemicontinuous at $x_{0}$;

(b) The function $\left(x^{*}, x\right) \mapsto \phi\left(x^{*}, x\right)-\phi\left(x^{*}, x_{0}\right)$ is convex in $x$ and upper semicontinuous, concave in $x^{*}$.

Proof: Let $T_{1}$ be a suboperator mentioned in (a). Define an operator $T_{2}$ on $K$ by

$$
T_{2}(x)= \begin{cases}T_{1}(x) & \text { if } x \in K \cap U \\ T(x) & \text { if } x \notin K \cap U .\end{cases}
$$

Then $x_{0}$ is a local solution of problem $(M)$ with $T_{2}$ instead of $T$ because $T_{2}$ is a suboperator of $T$. According to Proposition 3.1, $x_{0}$ is a solution of problem $\left(V_{w}\right)$ for $T_{2}$. By Proposition 3.2 , it is also a solution of problem $\left(V_{0}\right)$ for $T_{2}$, hence for $T$ as well.

It is worthwhile noticing that when $K$ is a nonempty and convex set in a locally convex space, Proposition 3.2 and Corollary 3.3 remain true if $\phi$ is lower semicontinuous, quasiconvex (instead of being convex) in $x$.

\section{EXISTENCE OF SOLUTIONS}

We are now ready to establish sufficient conditions for existence of solutions of the variational inequality models described in Section 1 with relatively simple proofs. Recall that a real-valued function $f$ on $K$ is said to be quasiconvex if for $x, y \in K$ and $t \in(0,1)$ one has $f(t x+(1-t) y) \leqslant \max \{f(x), f(y)\}$. It is said to be semistrictly quasiconvex (respectively, strictly quasiconvex) if $f(t x+(1-t) y)<\max \{f(x), f(y)\}$ whenever $f(x) \neq f(y)$ (respectively, $x \neq y$ ).

The following proposition provides an existence result for problem $(M)$. If $K$ is nonempty, convex and compact and if the operator $T$ is nonempty valued on $K$ then this proposition can be derived from [3, Proposition 2.1] by considering the real valued function $f$ on $K \times K$ defined by $f(x, y):=\sup _{x^{*} \in T(x)}\left(\phi\left(x^{*}, y\right)-\phi\left(x^{*}, x\right)\right)$.

Although the proof of Proposition 4.1 uses quite standard arguments we include it here since no assumption of nonemptiness is made on the value of the operator $T$.

Proposition 4.1. Problem $(M)$ has a solution if the following conditions hold:

(a) $K$ is nonempty convex and closed;

(b) $\phi$ is lower semicontinuous in $x$ on $K$;

(c) $T$ is properly $\phi$-quasimonotone on $K$;

(d) There is a compact set $K_{0} \subseteq K$ and $x_{0} \in K_{0}$ such that for every $x \in K \backslash K_{0}$ one has $\phi\left(x_{0}^{*}, x\right)-\phi\left(x_{0}^{*}, x_{0}\right)>0$ for some $x_{0}^{*} \in T\left(x_{0}\right)$.

Moreover, if additionally $\phi$ is quasiconvex in $x$, then the solution set of $(M)$ is closed, convex and included in $K_{0}$. 
Proof: We consider the following set-valued map $G$ from $K$ to itself:

$$
G(x):=\left\{y \in K: \phi\left(x^{*}, x\right)-\phi\left(x^{*}, y\right) \geqslant 0, \forall x^{*} \in T(x)\right\} .
$$

By hypothesis, $G(x)$ is nonempty and closed. It follows from (d) that $G\left(x_{0}\right)$ is a compact set. Moreover, $G$ is a KKM map in the sense that for $x_{1}, \ldots, x_{n} \in K$ one has $\operatorname{co}\left\{x_{1}, \ldots, x_{n}\right\} \subseteq \bigcup_{i=1}^{n} G\left(x_{i}\right)$, which is immediate from the definition of proper $\phi$-quasimonotonicity. By applying Ky Fan's theorem [6] to this map, we have $\bigcap_{x \in K} G(x)$ $\neq \emptyset$. Any element of this intersection is a solution of problem $(M)$. It is clear that any solution of $(M)$ belongs to the intersection, hence to $G\left(x_{0}\right) \subseteq K_{0}$. The convexity and the closeness of the solution set are immediate from condition (b) and the quasiconvexity of $\phi$ in the second variable.

It turns out that if in the model $(M)$ one sets $Y=K, \phi=f$ and $T(x)=x$ for $x \in K$, then one obtains a dual equilibrium problem of type

$(D E 1) \quad$ Find $x_{0} \in K$ such that $f\left(x, x_{0}\right) \leqslant f(x, x)$ for all $x \in K$.

In general, each solution of $(D E 1)$ is a solution of the dual equilibrium problem considered in [3], and the converse is not true. Hence the conclusion of Proposition 4.1 is stronger than the corresponding one of [3] (under a bit stronger hypothesis). In the case when $f(x, x)=0$ for $x \in K$, these results are equivalent.

It is interesting to note that in the above proposition proper $\phi$-quasimonotonicity can not be replaced by $\phi$-quasimonotonicity even when $\phi$ is the pairing function $\langle.,$.$\rangle and T$ is a single-valued and continuous operator from $K$ to $X^{\prime}$ (see $\left.[12,17]\right)$. The argument of [12] can be adapted to show the equivalence between the existence of solutions of problem $(M)$ on compact subsets of $K$ and the proper $\phi$-quasimonotonicity of $T$ on $K$. Namely the following corollary is an extension of [12, Theorem 1] to our model. The remark we have made after Proposition 4.1 is also available for this corollary in comparison with [ 3 , Theorem 2.1] on the dual equilibrium problem.

Corollary 4.2. Assume that $\phi$ is lower semicontinuous and quasiconvex in $x$ on $K$. Then the following conditions are equivalent:

(a) $T$ is properly $\phi$-quasimonotone on $K$;

(b) The set-valued map $G$ defined in the proof of Proposition 4.1 is KKM on $K$;

(c) For every nonempty convex and compact set $D \subseteq K$, problem $(M)$ has a solution on $D$;

(d) For every closed convex set $D \subseteq K$ if there are some compact set $D_{0} \subseteq D$ and $x_{0} \in D_{0}$ such that for every $x \in D \backslash D_{0}$ one has $\phi\left(x_{0}^{*}, x\right)-\phi\left(x_{0}^{*}, x_{0}\right)>0$ for some $x_{0}^{*} \in T\left(x_{0}\right)$, then problem $(M)$ has a solution on $D$.

Proof: The equivalence between (a) and (b) is obvious. Moreover, according to Proposition 4.1, (a) implies (d), while (d) obviously implies (c). So it remains to prove 
that (b) follows from (c). We do it by induction on the number $n$ in the inclusion $\operatorname{co}\left\{x_{1}, \ldots, x_{n}\right\} \subseteq \bigcup_{i=1, \ldots, n} G\left(x_{i}\right)$. The conclusion is evident when $n=1$. Assuming that it is true for any $n-1$ points of $K$, we consider $x_{1}, \ldots, x_{n} \in K$. Denote by $D=\operatorname{co}\left\{x_{1}, \ldots, x_{n}\right\}$ and by $y \in D$ a solution of $(M)$ on $D$ which exists by condition (c). Let $x \in D$. If $x=y$, there is nothing to prove. If $x \neq y$, then the ray starting from $y$ and going through $x$ meets the relative boundary of $D$ at some point $z$. By induction there is some $1 \leqslant k \leqslant n$ such that $z \in G\left(x_{k}\right)$. Since $y \in G\left(x_{k}\right)$ and $G\left(x_{k}\right)$ is convex, we derive that $x \in[y, z] \subseteq G\left(x_{k}\right) \subseteq \bigcup_{i=1, \ldots, n} G\left(x_{i}\right)$ which shows that $G$ is KKM.

For variational inequality models, as solutions of problem $\left(V_{0}\right)$ can be obtained from those of $\left(V_{w}\right)$ by Proposition 3.2, we shall focus on problems $(V)$ and $\left(V_{w}\right)$ only. The next lemma is a generalisation of Aussel and Hadjisavvas' recent result ([2, Proposition 2.1]) which shows that under a continuity hypothesis, a quasimonotone operator is properly quasimonotone whenever the associated Minty problem has no local solutions.

Lemma 4.3. Assume that $K$ is nonempty and convex, and that $\phi$ is lower semicontinuous and quasiconvex in $x$ on $K$. If $T$ is $\phi$-quasimonotone on $K$, then either it is properly $\phi$-quasimonotone, or problem $(M)$ has a local solution.

Proof: Suppose that $T$ is not properly $\phi$-quasimonotone. There exist $x_{1}, \ldots, x_{n}$ $\epsilon K$ and a convex combination $x$ of these points such that, for any $i=1, \ldots, n, \phi\left(x_{i}^{*}, x\right)$ $-\phi\left(x_{i}^{*}, x_{i}\right)>0$ for some $x_{i}^{*} \in T\left(x_{i}\right)$. Since $\phi$ is lower semicontinuous in the second variable, there is a neighbourhood $U$ of $x$ such that for any $i=1, \ldots, n, \phi\left(x_{i}^{*}, y\right)$ $-\phi\left(x_{i}^{*}, x_{i}\right)>0$ for every $y \in K \cap U$. It follows from the $\phi$-quasimonotonicity of $T$ that $\phi\left(y^{*}, y\right)-\phi\left(y^{*}, x_{i}\right) \geqslant 0$ for every $y^{*} \in T(y), y \in K \cap U$ and $i=1, \ldots, n$. The quasiconvexity of $\phi$ yields $\phi\left(y^{*}, y\right)-\phi\left(y^{*}, x\right) \geqslant 0$ for all $y^{*} \in T(y), y \in K \cap U$. Thus, $x$ is a local solution of problem $(M)$.

THEOREM 4.4. Problem $(V)$ has a solution if the following conditions hold:

(a) $K$ is nonempty, convex and closed;

(b) $\phi$ is lower semicontinuous and quasiconvex in $x$ on $K$;

(c) $T$ is $\phi$-quasimonotone and $\phi$-hemicontinuous;

(d) There is a compact set $K_{0} \subseteq K$ and $x_{0} \in K_{0}$ such that for every $x \in K \backslash K_{0}$ one has $\phi\left(x_{0}^{*}, x\right)-\phi\left(x_{0}^{*}, x_{0}\right)>0$ for some $x_{0}^{*} \in T\left(x_{0}\right)$.

In addition, if either $T$ is weakly $\phi$-pseudomonotone or $\phi$ is strictly quasiconvex in $x$, then the solution set of $(V)$ is included in $K_{0}$.

Proof: The first part of the proposition is obtained from Lemma 4.3, Propositions 3.1(ii) and 4.1. To prove the second part, let $x \in K \backslash K_{0}$. If it was a solution of $(V)$, then (d) would imply $\phi\left(x^{*}, x\right)-\phi\left(x^{*}, x_{0}\right)=0$ for every $x^{*} \in T(x)$. When $T$ is weakly $\phi$-pseudomonotone, we derive that $\phi\left(x_{0}^{*}, x_{0}\right)-\phi\left(x_{0}^{*}, x\right) \geqslant 0$ which is a contradiction with 
(d). When $\phi$ is strictly quasiconvex, for $z$ between $x$ and $x_{0}$, we have $\phi\left(x^{*}, x\right)-\phi\left(x^{*}, z\right)$ $>0$, which is again a contradiction with the assumption that $x$ is a solution.

Proposition 4.5. Problem $\left(V_{w}\right)$ has a solution if the following conditions hold:

(a) $K$ is nonempty, convex and closed;

(b) $\phi$ is lower semicontinuous and quasiconvex in $x$ on $K$;

(c) $T$ is $\phi$-quasimonotone on $K$ and locally admits a weakly $\phi$-hemicontinuous suboperator;

(d) There is a compact set $K_{0} \subseteq K$ and $x_{0} \in K_{0}$ such that for every $x \in K \backslash K_{0}$ one has $\phi\left(x_{0}^{*}, x\right)-\phi\left(x_{0}^{*}, x_{0}\right)>0$ for some $x_{0}^{*} \in T(x)$.

In addition, if either $T$ is weakly $\phi$-pseudomonotone or $\phi$ is strictly quasiconvex in $x$, then the solution set of $\left(V_{w}\right)$ is included in $K_{0}$.

ProOF: Apply Lemma 4.3, Propositions 3.1(iii) and 4.1 to obtain the first part of the proposition. For the second part, use the same technique as the proof of the preceding theorem.

We observe that unlike the model $(M)$, the solution set of $(V)$ is not necessarily bounded when $T$ is properly quasimonotone and $\phi$ is quasiconvex in $x$, as seen by the next example.

EXAMPLE 4.6. Consider problem $(V)$ with $X=Y=\mathbb{R}^{n}, K=\mathbb{R}_{+}^{n}, \phi\left(x^{*}, x\right)=\left\langle x^{*}, x\right\rangle$ and $T$ is given by

$$
T(x)= \begin{cases}K & \text { if } x=0 \\ \{0\} & \text { else. }\end{cases}
$$

Direct verification shows that conditions (a) through (d) of Theorem 4.4 are true. Despites of this, the solution set of this problem is the unbounded set $K$. In this example $T$ is properly quasimonotone, but not pseudomonotone, and $\phi$ is linear, but not strictly quasiconvex in $x$.

In the remaining of this section we assume that $X$ is a locally convex space. In this context milder coercivity conditions can be developed to ensure the existence of solutions of variational inequalities. We recall that a set $A \subseteq X$ is said to be locally compact if for every $x \in X$ there is a neighbourhood $U$ of $x$ such that $U \cap A$ is a compact set.

PROPOSITION 4.7. Problem $(V)$ has a solution if the following conditions hold:

(a) $K$ is nonempty, convex and locally compact;

(b) $\phi$ is lower semicontinuous and semistrictly quasiconvex in $x$ on $K$;

(c) $T$ is $\phi$-quasimonotone and $\phi$-hemicontinuous;

(d) There is a compact set $K_{0} \subseteq K$ such that for every $x \in K \backslash K_{0}$ one can find $x_{0} \in K_{0}$ with $\phi\left(x^{*}, x\right)-\phi\left(x^{*}, x_{0}\right) \geqslant 0$ for all $x^{*} \in T(x)$. 
Moreover, condition (d) is also necessary for problem $(V)$ to have a solution provided that $T$ is $\phi$-pseudomonotone.

Proof: Let us take any point $a \in K$. By definition, there is a convex neighbourhood $V$ of the origin such that the intersection of $a+V$ with $K$ is compact (and nonempty). We may suppose that $V$ is a closed neighbourhood. For $t>1$ sufficiently large, $a+t V$ is a neighbourhood of the origin. Moreover, the intersection of this neighbourhood with $K$ is compact, because

$$
[(a+t V) \cap K] \subseteq a+t[((a+V) \cap K)-a] .
$$

Set $U=a+t(i n t V)$. It is a convex open neighbourhood of the origin whose intersection with $K$ is nonempty and relatively compact. As $K_{0}$ is compact, there is a positive $n \geqslant 1$ such that $K_{0} \subseteq(n U) \cap K$. Consider the closure $K_{n}$ of the set $(n U) \cap K$. This set is nonempty and convex. It is also compact because when $t>0$ is sufficiently small, one has $a+t\left(K_{n}-a\right) \subset(V \cap K)$, which implies that the set $a+t\left(K_{n}-a\right)$ is compact, hence so is $K_{n}$. By Theorem 4.4, the problem $(V)$ on $K_{n}$ admits a solution $y_{0}$. If $y_{0} \in K_{0}$, we are done. If not, in view of (d), there is $x_{0} \in K_{0}$ such that $\phi\left(y_{0}^{*}, y_{0}\right)-\phi\left(y_{0}^{*}, x_{0}\right) \geqslant 0$ for every $y_{0}^{*} \in T\left(y_{0}\right)$. Actually this inequality is equality because $y_{0}$ is a solution of $(V)$ on $K_{n}$. For every $y \in K \backslash\left\{x_{0}\right\}$, there is $t \in(0,1)$ such that $x_{0}+t\left(y-x_{0}\right) \in K_{n}$. Since $\phi$ is semistrictly quasiconvex, we have either $\phi\left(y_{0}^{*}, x_{0}\right)=\phi\left(y_{0}^{*}, y\right)$ or

$$
\phi\left(y_{0}^{*}, x_{0}+t\left(y-x_{0}\right)\right)<\max \left(\phi\left(y_{0}^{*}, x_{0}\right), \phi\left(y_{0}^{*}, y\right)\right)
$$

which leads to $\phi\left(y_{0}^{*}, y\right) \geqslant \phi\left(y_{0}^{*}, y_{0}\right)$ for every $y_{0}^{*} \in T\left(y_{0}\right)$. By this $y_{0}$ is a solution of problem $(V)$.

When $T$ is $\phi$-pseudomonotone, by setting $K_{0}=\left\{x_{0}\right\}$ where $x_{0}$ is any solution of problem $(V)$ we deduce condition (d).

Corollary 4.8. Under conditions (a), (b) and (c) of Proposition 4.7, the following condition is sufficient for problem $(V)$ to have a nonempty and bounded solution set

(d') There is a compact set $K_{0} \subseteq K$ such that for every $x \in K \backslash K_{0}$ one can find $x_{0} \in K_{0}$ with $\phi\left(x^{*}, x\right)-\phi\left(x^{*}, x_{0}\right)>0$ for all $x^{*} \in T(x)$.

This condition is also a necessary condition provided that $\phi$ is strictly quasiconvex in $x$ and $T$ is $\phi$-pseudomonotone.

Proof: Under the hypothesis of this corollary, Proposition 4.7 shows that the solution set of problem $(V)$ is nonempty. Moreover, condition (d) shows that all solutions of $(V)$ belong to $K_{0}$, hence the solution set is nonempty and bounded. Conversely, assume that the solution set of $(V)$ is nonempty and bounded. Let $K_{0}$ be its closure which is compact. For every $x \in K \backslash K_{0}$, we have $\phi\left(x_{0}^{*}, x\right)-\phi\left(x_{0}^{*}, x_{0}\right) \geqslant 0$ for every $x_{0} \in K_{0}, x_{0}^{*} \in T\left(x_{0}\right)$ which implies $\phi\left(x^{*}, x\right)-\phi\left(x^{*}, x_{0}\right) \geqslant 0$ for every $x_{0} \in K_{0}, x^{*} \in T(x)$. 
If equality holds for some $x_{0} \in K_{0}$ and $x^{*} \in T(x)$, then due to the $\phi$-pseudomonotonicity of $T$ one has $\phi\left(x_{0}^{*}, x_{0}\right)=\phi\left(x_{0}^{*}, x\right)$ for all $x_{0}^{*} \in T\left(x_{0}\right)$. One arrives at a contradiction because $\phi\left(x_{0}^{*}, z\right)-\phi\left(x_{0}^{*}, x_{0}\right)<0$ for all $z$ between $x_{0}$ and $x$ due to the strict quasiconvexity of $\phi$.

Proposition 4.9. Problem $\left(V_{w}\right)$ has a solution if the following conditions hold:

(a) $K$ is nonempty, convex and locally compact;

(b) $\phi$ is lower semicontinuous and semistrictly quasiconvex in $x$ on $K$;

(c) $T$ is $\phi$-quasimonotone on $K$ and locally admits a weakly $\phi$-hemicontinuous suboperator;

(d) There is a compact set $K_{0} \subseteq K$ such that for every $x \in K \backslash K_{0}$ one can find $x_{0} \in K_{0}$ with $\phi\left(x^{*}, x\right)-\phi\left(x^{*}, x_{0}\right) \geqslant 0$ for all $x^{*} \in T(x)$.

Moreover, condition (d) is also necessary for problem $(V)$ to have a solution provided that $T$ is $\phi$-pseudomonotone.

Proof: Use the same argument as that of the proof of Proposition 4.7.

Corollary 4.10. Under conditions (a), (b) and (c) of Proposition 4.9, the following condition is sufficient for problem $\left(V_{w}\right)$ to have a nonempty and bounded solution set

(d') There is a compact set $K_{0} \subseteq K$ such that for every $x \in K \backslash K_{0}$ one can find $x_{0} \in K_{0}$ with $\phi\left(x^{*}, x\right)-\phi\left(x^{*}, x_{0}\right)>0$ for all $x^{*} \in T(x)$.

This condition is also a necessary condition provided that $\phi$ is strictly quasiconvex in $x$ and $T$ is $\phi$-pseudomonotone.

ProOF: By the same technique as Corollary 4.8.

To conclude we notice that the results of this section generalise several existence conditions given in recent works $[2,4,5,9,17,21,25,27]$ and some others for mixed variational inequalities with set-valued pseudomonotone operators and for standard variational inequalities with set-valued quasimonotone operators. For instance, by setting $Y=X^{\prime}$ and $\phi\left(x^{*}, x\right)=\left\langle x^{*}, x\right\rangle,[5$, Theorem 5.1], [9, Theorem 3.1], [17, Theorem 4.1, 4.2], [21, Theorem 4.3] are derived from Corollary 4.10 while a theorem analogous to [2, Theorem 2.1] can be obtained from Proposition 4.5 and Corollary 3.3 as a special case; [4, Theorem 4.5], [25, Theorem 3] are obtained from Theorem 4.4 by setting $Y=X^{\prime}$ and $\phi\left(x^{*}, x\right)=\left\langle x^{*}, x\right\rangle+h(x)$ where $h$ is a lower semicontinuous convex function on $X$ (actually this particular case of Theorem 4.4 gives a stronger result than the corresponding one of [4] and [25] because of using quasimonotonicity instead of pseudomonotonicity). Finally, complementarity models in which the pairing function $\langle.,$.$\rangle is replaced by a cou-$ pling function $\phi$ can also be studied and existence results can be obtained. For instance, 
let us consider the problem of finding $x_{0} \in K$ such that

$$
\phi\left(x_{0}^{*}, x_{0}\right)=0 \text { for all } x_{0}^{*} \in T\left(x_{0}\right) .
$$

The next result is immediate from Theorem 4.4.

COROLlary 4.11. Assume that the following conditions hold

(i) $K$ is a convex and closed cone;

(ii) $\phi$ is lower semicontinuous and linear in $x$;

(iii) $T$ is $\phi$-quasimonotone, $\phi$-hemicontinuous;

(iv) There exist a compact set $K_{0} \subseteq K$ and $x_{0} \in K_{0}$ such that for all $x \in K \backslash K_{0}$, one has $\phi\left(x_{0}^{*}, x-x_{0}\right)>0$, for some $x_{0}^{*} \in T\left(x_{0}\right)$.

Then the complementarity problem has a solution.

The counterparts of Theorem 4.5, Propositions 4.7 and 4.9 for the complementarity problem are derived in a similar way.

\section{Parametric equilibrium PROBlems}

Let us now consider the parametric equilibrium problem already mentioned in the introduction:

(E) Find $x_{0} \in K$ such that

$$
f\left(x_{0}, x\right)+h(z, x)-h\left(z, x_{0}\right) \geqslant 0, \forall x \in K, z \in Z,
$$

where $Z$ is a nonempty set, $f$ is a real-valued function on $K \times K$ with $f(x, x)=0$ for every $x \in K$ and $h$ is a real-valued function on $Z \times K$. The equilibrium model studied in [7] is a particular case of problem $(E)$ when $Z=K$ and inequality is required for $z=x_{0}$ only.

The equilibrium problem $(D)$ is clearly a particular case of $(E)$ and is covered by model $(V)$. It is to note that conversely, by suitably choosing the function $f$ and $h$ we can also derive models $(V)$ and $(M)$ from this equilibrium problem $(D)$. Indeed, by taking $h=0$ and respectively

$$
f(x, y)=\inf _{x^{*} \in T(x)}\left(\phi\left(x^{*}, y\right)-\phi\left(x^{*}, x\right)\right)
$$

and

$$
f(x, y)=\inf _{x^{*} \in T(y)}\left(\phi\left(x^{*}, y\right)-\phi\left(x^{*}, x\right)\right)
$$

we obtain the models $(V)$ and $(M)$. A model a bit more general than $\left(V_{w}\right)$ can also be deduced from the equilibrium problem with $h=0$ and $f(x, y)=\sup _{x^{*} \in T(x)}\left(\phi\left(x^{*}, y\right)\right.$ $\left.-\phi\left(x^{*}, x\right)\right)$. This mutuality between variational inequality formulations and equilibrium formulations are useful in deriving results for one from the other. 
Proposition 5.1. Problem $(E)$ has a solution if the following conditions hold:

(a) $K$ is nonempty and convex;

(b) For every $y \in K$ and $z \in Z$, the function $x \mapsto f(y, x)+h(z, x)$ is lower semicontinuous and semistrictly quasiconvex on $K$;

(c) For every $x, y \in K$, strict inequality $f(y, x)+h\left(z_{0}, x\right)-h\left(z_{0}, y\right)>0$ for some $z_{0} \in Z$ implies $h(z, x)-h(z, y)-f(x, y) \geqslant 0$ for all $z \in Z$;

(d) $h$ is lower semicontinuous in $x$ on $K$ and for every $x, y \in K$ the function $t \in[0,1] \mapsto f(x+t(y-x), y)$ is upper semicontinuous at $t=0$;

(e) There is a compact set $K_{0} \subseteq K$ and $x_{0} \in K_{0}$ such that for every $x \in K \backslash K_{0}$ one has $f\left(x_{0}, x\right)+h(z, x)-h\left(z, x_{0}\right)>0$ for every $z \in Z$.

Moreover, the solution set of problem $(E)$ is included in $K_{0}$ if additionally either of the following conditions holds:

( $\left.\mathrm{b}^{\prime}\right)$ For every $y \in K$ and $z \in Z$, the function $x \mapsto f(y, x)+h(z, x)$ is lower semicontinuous and strictly quasiconvex on $K$;

(c) For every $x, y \in K$, inequality $f(y, x)+h\left(z_{0}, x\right)-h\left(z_{0}, y\right) \geqslant 0$ for some $z_{0} \in Z$ implies $h(z, x)-h(z, y)-f(x, y) \geqslant 0$ for all $z \in Z$.

Proof: We wish to apply Theorem 4.4 to this problem. Remember that $(E)$ can be written in form of $(V)$ by setting

$$
Y=K \times Z, T(x)=\{x\} \times Z
$$

and

$$
\phi((y, z), x)=f(y, x)+h(z, x)
$$

for every $(y, z) \in Y$ and $x \in K$. We observe that conditions (a) and (b) of Theorem 4.4 are proved obviously, and condition (c) of the present proposition shows that $T$ is $\phi$-quasimonotone. We now prove that $T$ is $\phi$-hemicontinuous. Let $x, x_{0} \in K$ verifying $h\left(z, x_{t}\right)-f\left(x_{t}, x_{0}\right)-h\left(z, x_{0}\right) \geqslant 0$ for all $z \in Z$ and for $t>0$ sufficiently close to 0 , where $x_{t}=x_{0}+t\left(x-x_{0}\right)$. Then due to condition (b), $h\left(z, x_{t}\right) \leqslant f\left(x_{t}, x\right)+h(z, x)$ for every $z \in Z$. In view of condition (d) this yields $f\left(x_{0}, x\right)+h(z, x)-h\left(z, x_{0}\right) \geqslant 0$ for all $z \in Z$. By this $T$ is $\phi$-hemicontinuous and Theorem 4.4 yields the first part of the proposition. For the second part, it suffices to observe that condition (c') shows that $T$ is $\phi$-pseudomonotone.

When $X$ is a locally convex space the coercivity hypothesis (e) can be relaxed as in Proposition 4.7.

Proposition 5.2. Assume that $X$ is a locally convex space. Problem $(E)$ has a solution if the following conditions hold:

(a) $K$ is nonempty, convex and locally compact; 
(b) For every $y \in K$ and $z \in Z$, the function $x \mapsto f(y, x)+h(z, x)$ is lower semicontinuous and semistrictly quasiconvex on $K$;

(c) For every $x, y \in K$, strict inequality $f(y, x)+h\left(z_{0}, x\right)-h\left(z_{0}, y\right)>0$ for some $z_{0} \in Z$ implies $h(z, x)-h(z, y)-f(x, y) \geqslant 0$ for all $z \in Z$;

(d) $h$ is lower semicontinuous in $x$ on $K$ and for every $x, y \in K$ the function $t \in[0,1] \mapsto f(x+t(y-x), y)$ is upper semicontinuous at $t=0$;

(e) There is a compact set $K_{0} \subseteq K$ such that for every $x \in K \backslash K_{0}$ one can find $x_{0} \in K_{0}$ with $h(z, x)-f\left(x, x_{0}\right)-h\left(z, x_{0}\right) \geqslant 0$ for every $z \in Z$.

Moreover, condition (e) is also a necessary condition when (a), (b), (d) and the following condition holds

(c') For every $x, y \in K$, inequality $f(y, x)+h\left(z_{0}, x\right)-h\left(z_{0}, y\right) \geqslant 0$ for some $z_{0} \in Z$ implies $h(z, x)-h(z, y)-f(x, y) \geqslant 0$ for all $z \in Z$;

Proof: Invoke the proof of Proposition 5.1 and Proposition 4.7. Furthermore, condition (c') shows that $T$ is $\phi$-pseudomonotone. Hence Proposition 4.7 is applicable.

The boundedness of the solution set can also be assured under stronger conditions.

Corollary 5.3. Under conditions (a), (b), (c) and (d) of Proposition 5.2, the following condition is sufficient for problem $(E)$ to have a nonempty and bounded solution set

$\left(\mathrm{e}^{\prime}\right)$ There is a compact set $K_{0} \subseteq K$ such that for every $x \in K \backslash K_{0}$ one can find $x_{0} \in K_{0}$ with $h(z, x)-f\left(x, x_{0}\right)-h\left(z, x_{0}\right)>0$ for every $z \in Z$.

It is also a necessary condition provided that

(b') For every $y \in K$ and $z \in Z$, the function $x \mapsto f(y, x)+h(z, x)$ is lower semicontinuous and strictly quasiconvex on $K$;

(c') For every $x, y \in K$, inequality $f(y, x)+h\left(z_{0}, x\right)-h\left(z_{0}, y\right) \geqslant 0$ for some $z_{0} \in Z$ implies $h(z, x)-h(z, y)-f(x, y) \geqslant 0$ for all $z \in Z$;

Proof: Invoke to Corollary 4.8.

We notice that Proposition 5.2 provides a strengthened version of $[7$, Theorem 4.3] for two reasons. Firstly, in [7] condition that $f(x, y)+f(y, x) \leqslant 0$ is required which is stronger than conditions (c) and ( $\left.c^{\prime}\right)$. Secondly, in [7], it is considered only the case $Z=K$ and the inequality in $(E)$ is required to hold for some $z \in K$ only; thus the solutions obtained by Proposition 5.2 are stronger than those of the model of [7].

\section{FRICTIONLESS CONTACT PROBLEM}

In this section we apply our results to the Signorini frictionless contact problem (see $[15,11]$, for example). The classical way to obtain existence results for contact problems is to assume some strong monotonicity and Lipschitz continuity of the elasticity operator. 
The results of Section 4 enable us to derive existence criteria for this problem under weaker conditions.

To facilitate the reading, we follow the notations of the above references. Let $\Omega$ be an open, bounded and connected region in $\mathbb{R}^{k}$ with $k=1,2$, or 3 , which represents the interior of an elastic body. Let $\Gamma$ be its boundary which is assumed to be Lipschitz and partitioned into three parts $c l\left(\Gamma_{1}\right), c l\left(\Gamma_{2}\right)$ and $c l\left(\Gamma_{3}\right)$. It is supposed that $\Gamma_{1}, \Gamma_{2}$ and $\Gamma_{3}$ are disjoint, $\Gamma_{1}$ is fixed and of strictly positive measure in $\Gamma$. The body is fixed on $\Gamma_{1}$ and is in a frictionless contact with a fixed foundation on $\Gamma_{3}$. In the model proposed by Signorini that we are considering it is assumed that surface tractions of density $f_{2} \in\left[L^{2}\left(\Gamma_{2}\right)\right]^{k}$ act on $\Gamma_{2}$ and volume forces of density $f_{0} \in\left[L^{2}(\Omega)\right]^{k}$ act on $\Omega$. We shall make use of the following notations

$$
\begin{aligned}
& \mathbb{S}^{k}=\left\{\sigma=\left(\sigma_{i j}\right)_{i j} \in \mathbb{R}^{k \times k}: \sigma_{i j}=\sigma_{j i}\right\}=\mathbb{R}_{s}^{k \times k} \\
& W=\left\{v \in H^{1}(\Omega)^{k}: v=0 \text { on } \Gamma_{1}\right\} \\
& Q=\left\{q=\left(q_{i j}\right) \in L^{2}(\Omega)^{k \times k}: q_{i j}=q_{j i}, 1 \leqslant i, j \leqslant k\right\}=L^{2}(\Omega)_{s}^{k \times k} \\
& W_{2}=\left\{v \in W: v_{\nu} \leqslant 0 \text { almost everywhere on } \Gamma_{3}\right\}
\end{aligned}
$$

where $v_{\nu}$ is the normal component of $v$. In the sequel $\varepsilon$ stands for the (linear) deformation operator $\varepsilon: H^{1}(\Omega)^{k} \rightarrow Q$ defined by

$$
\varepsilon_{i j}(u)=\frac{1}{2}\left[\frac{\partial u_{i}}{\partial x_{j}}+\frac{\partial u_{j}}{\partial x_{i}}\right], \quad 1 \leqslant i, j \leqslant k .
$$

Equipped with the inner products

$$
\begin{gathered}
\langle p, q\rangle_{Q}=\int_{\Omega} p_{i j}(x) q_{i j}(x) d x \\
\langle u, v\rangle_{W}=\langle\varepsilon(u), \varepsilon(v)\rangle_{Q}
\end{gathered}
$$

the spaces $W$ and $Q$ are real Hilbert spaces and $W_{2}$ is a nonempty closed convex set of $W$ (see [11] for example).

Let $\mathcal{F}: \Omega \times \mathbb{S}^{k} \rightarrow \mathbb{S}^{k}$ be a given elasticity operator. We define the stress function $\sigma: H^{1}(\Omega)^{k} \rightarrow Q$ which associate to any vector-valued function $u: \Omega \rightarrow \mathbb{R}^{k}$ of $H^{1}(\Omega)^{k}$ its stress field defined by

$$
\begin{aligned}
\sigma(u): \Omega & \rightarrow \mathbb{S}^{k} \\
x & \mapsto \mathcal{F}(x, \varepsilon(u)(x)) .
\end{aligned}
$$

With the above notations the equilibrium problem of this elastic and frictionless contact can be formulated as follows. 
Find a displacement field $u: \Omega \rightarrow \mathbb{R}$ such that

$$
\begin{aligned}
-\operatorname{Div} \sigma(u) & =f_{0} & & \text { in } \Omega \\
u & =0 & & \text { on } \Gamma_{1} \\
\sigma(u) \nu & =f_{2} & & \text { on } \Gamma_{2} \\
u_{\nu} \leqslant 0, \sigma(u)_{\nu} \leqslant 0, \sigma(u)_{\nu} u_{\nu} & =0, \sigma(u)_{\tau}=0 & & \text { on } \Gamma_{3}
\end{aligned}
$$

where $\nu$ is the unit outward normal vector on $\Gamma$ while $\sigma_{\nu}$ and $\sigma_{\tau}$ denotes respectively the normal and the tangential components of $\sigma$.

Following the classical approach of Stampacchia an variational problem can be formulated:

$(\widetilde{P}) \quad$ Find $u \in W_{2}$ such that $\langle\sigma(u), \varepsilon(v)-\varepsilon(u)\rangle_{Q} \geqslant\langle f, v-u\rangle_{W}, \quad \forall v \in W_{2}$ where $f$ denotes an element of $W$ defined by

$$
\langle f, v\rangle_{W}=\int_{\Omega} f_{0} \cdot v d x+\int_{\Gamma_{2}} f_{2} \cdot v d a .
$$

As in $([15])$ it can be shown that any solution of $(P)$ (element of $W_{2}$ ) is also a solution of $(\widetilde{P})$. The converse is true if the solution found for $(\widetilde{P})$ is an element of $\mathcal{C}^{2}(\Omega)$. Otherwise a solution of $(\widetilde{P})$ is a weak solution of $(P)$ in a sense described in [15, Theorem 6.3].

Let us define the operator $\tilde{\phi}: Q \times W \rightarrow \mathbb{R}$ by

$$
\widetilde{\phi}(q, v)=\langle q, \varepsilon(v)\rangle_{Q}-\langle f, v\rangle_{W} .
$$

Proposition 6.1. Assume that the stress function satisfies the following properties

(i) the map $\sigma$ is $\tilde{\phi}$-quasimonotone and $\tilde{\phi}$-hemicontinous on $W_{2}$.

(ii) there exist a compact subset $W_{0}$ of $W_{2}$ and a function $u_{0}$ of $W_{0}$ such that

$$
\left.\left\langle\sigma\left(u_{0}\right), \varepsilon(u)-\varepsilon\left(u_{0}\right)\right\rangle_{Q}\right\rangle\left\langle f, u-u_{0}\right\rangle_{W} \text { for every } u \in W_{2} \backslash W_{0} .
$$

Then the variational contact problem $(\widetilde{P})$ admits a solution.

ProOF: By applying the Riez representation theorem we may define an operator $A: W_{2} \rightarrow W_{2}$ such that

$$
\langle A(u), v\rangle_{W}=\langle\sigma(u), \varepsilon(v)\rangle_{Q}, \quad \forall u, v \in W_{2} .
$$

Now the variational problem $(\widetilde{P})$ corresponds to the model $(V)$ of Section 1 by considering $X=Y=W, K=W_{2}$, with $\phi: W \times W_{2} \rightarrow \mathbb{R}$ and $T: W_{2} \rightarrow W$ defined by

$$
\phi(u, v)=\langle u-f, v\rangle_{W} \quad \text { and } \quad T(u)=A(u) .
$$


We notice as above that the subset $W_{2}$ is nonempty convex and closed in $W$. The operator $\phi$ is clearly continuous and linear with respect to the second variable. Moreover hypothesis $d$ ) of Theorem 4.4 is satisfied since, from (ii), there exists $W_{0} \subset W_{2}$ compact and $u_{0} \in W_{0}$ such that, for any $u \in W \backslash W_{2}$,

$$
\phi\left(T\left(u_{0}\right), u\right)-\phi\left(T\left(u_{0}\right), u_{0}\right)=\left\langle\sigma\left(u_{0}\right), \varepsilon(u)-\varepsilon\left(u_{0}\right)\right\rangle_{Q}-\left\langle f, u-u_{0}\right\rangle_{W}>0 .
$$

Thus, in order to apply Theorem 4.4 it is now sufficient to observe that the $\widetilde{\phi}$-quasimonotonicity and the $\widetilde{\phi}$-hemicontinuity of the operator $\sigma$ implies the $\phi$-quasimonotonicity and the $\phi$-hemicontinuity of $T$ on $W_{2}$.

\section{REFERENCES}

[1] D. Aussel, 'Subdifferential properties of quasiconvex and pseudoconvex functions: unified approach', J. Optim. Theory Appl. 97 (1998), 29-45.

[2] D. Aussel and N. Hadjisavvas, 'On quasimonotone variational inequalities', J. Optim. Theory Appl. 121 (2004), 445-450.

[3] M. Bianchi and R. Pini, 'A note on equilibrium problems with properly quasimonotone bifunctions', J. Global Optim. 20 (2001), 67-76.

[4] M.S.R. Chowdhury and K.K. Tan, 'Generalized variational inequalities for quasimonotone operators and applications', Bull. Polish Acad. Sci. Math. 45 (1997), 25-54.

[5] A. Daniilidis and N. Hadjisavvas, 'Characterization of nonsmooth semistrictly quasiconvex and strictly quasiconvex functions', J. Optim. Theory Appl. 102 (1999), 525-536.

[6] K. Fan, 'Minimax theorems', Proc. Natl. Acad. Sci. USA 39 (1953), 42-47.

[7] F. Flores-Bazan, 'Existence theorems for generalized noncoercive equilibrium problems: the quasiconvex case', SIAM J. Optim. 11 (2000), 675-690.

[8] F. Giannessi and A. Maugeri, Variational inequalities and network equilibrium problems (Plenum Press, New York, 1995).

[9] N. Hadjisavvas and S. Schaible, 'Quasimonotone variational inequalities in Banach spaces', J. Optim. Theory Appl. 90 (1996), 95-111.

[10] P.T. Harker and J.S. Pang, 'Finite-dimensional variational inequality and nonlinear complementarity problem: a survey of theory, algorithms and applications', Math. Programming 48 (1990), 161-220).

[11] W. Han and M. Sofonea, Quasistatic contact problems in viscoelasticity and viscoplasticity, AMS/IP Studies in Advanced Mathematics 30 (American Mathematical Society, Providence, R.I., 2002).

[12] R. John, 'A note on Minty variational inequalities and generalized monotonicity', in Generalized Convexity and Generalized Monotonicity, (N. Hadjisavvas, J-E. Martinez-Legaz and J-P. Penot, Editors), Lecture Notes in Econom. and Math. Systems 502 (Springer, Berlin, 2001), pp. 240-246.

[13] S. Karamardian, 'Complementarity over cones with monotone and pseudomonotone maps', J. Optim. Theory Appl. 18 (1976), 445-454.

[14] S. Karamardian and S. Schaible, 'Seven kinds of monotone maps', J. Optim. Theory Appl. 66 (1990), 37-46. 
[15] N. Kikuchi and J.T. Oden, Contact problems in elasticity: Study of variational inequalities and finite element methods (SIAM, Philadelphia, 1988).

[16] D. Kinderlehrer and G. Stampacchia, An introduction to variational inequalities and their applications (Academic Press, New York, 1980).

[17] I. Konnov, 'On quasimonotone variational inequalities', J. Optim. Theory Appl. 99 (1998), 165-181.

[18] D.T. Luc, 'Characterizations of quasiconvex functions', Bull. Austral. Math. Soc. 48 (1993), 393-405.

[19] D.T. Luc, 'On generalized convex nonsmooth functions', Bull. Austral. Math. Soc. 49 (1994), 139-149.

[20] D.T. Luc, 'Generalized monotone maps and support bifunctions', Acta Math. Vietnam. 21 (1996), 213-252.

[21] D.T. Luc, 'Existence results for densely pseudomonotone variational inequalities', $J$. Math. Anal. Appl. 254 (2001), 291-308.

[22] M.A. Noor, 'Some recent advances in variational inequalities, Part I, basic concepts', New Zealand J. Math. 26 (1997), 53-80.

[23] M.A. Noor, 'Some recent advances in variational inequalities, Part II, other concepts', New Zealand J. Math. 26 (1997), 229-255.

[24] J-P. Penot and P.H. Quang, 'On generalized convexity of functions and generalized monotonicity of set-valued maps', J. Optim. Theory Appl. 92 (1997), 343-356.

[25] M.H. Shih and K.K. Tan, 'A further generalization of Ky Fan's minimax inequality and its applications', Studia Math. 78 (1984), 279-287.

[26] G. Stampacchia, 'Formes bilinéaires coercives sur les ensembles convexes', C. $R$. Acad. Sci. Paris 258 (1964), 4413-4416.

[27] J. C. Yao, 'Multivalued variational inequalities with K-pseudomonotone operators', $J$. Optim. Theory Appl. 83 (1994), 391-403.

[28] J.X. Zhou and G. Chen, 'Diagonally convex conditions for problems in convex analysis and quasi-variational inequalities', J. Math. Anal. Appl. 132 (1988), 213-225.

Lab. MANO

University of Perpignan

France

e-mail: aussel@univ-perp.fr
Lab. Analyse non linéaire et Géométrie

University of Avignon

France

e-mail: dtluc@univ-avignon.fr 